\title{
Fundamentos epistemológicos del liderazgo distribuido: el caso de la investigación en educación
}

\author{
DISTRIBUTED LEADERSHIP EPISTEMOLOGICAL BASES: THE CASE OF EDUCATION RESEARCH
}

\author{
Dr. Pablo López (pablopez@uchile.cl) Departamento de Educación, Universidad de Chile
}

\begin{abstract}
The article reviews research between 1999 and 2013 with the aim of knowing the epistemological foundations of research on distributed leadership, with special interest in education studies. We conclude it is an area in developing, with potential, but its empirical foundations are weak and methodological strategies are not always explicit, making it difficult to evaluate their findings somewhat. With respect to the theoretical basis, much of the research finds their sustenance in Engeström's activity theory and Hutchins's distributed cognition. The importance of distributed leadership studies is its growing influence in the administration of colleges and universities, making these studies, a major political issue.
\end{abstract}

Key words: distributed leadership, activity theory, distributed cognition theory, methodological strategies, research techniques.

\section{Resumen}

El artículo revisa investigaciones entre los años 1999 y 2013 con el objetivo de conocer las bases epistemológicas de la investigación en liderazgo distribuido, con especial interés en estudios de educación. Se concluye que es un campo en formación, con potencial, pero que sus bases empíricas son débiles y sus estrategias metodológicas no siempre están explícitas, lo que dificulta un poco evaluar sus descubrimientos. Con respecto a las bases teóricas, gran parte de las investigaciones encuentran su sustento en la teoría de la actividad de Engeström y la teoría de la cognición distribuida de Hutchins. La importancia de los estudios de liderazgo distribuido es su creciente influencia en la administración de colegios y universidades, lo que hace de estos estudios un tema de gran relevancia política.

Palabras clave: liderazgo distribuido, teoría de la actividad, teoría de la cognición distribuida, estrategias metodológicas, técnicas de investigación.

\section{Introducción}

Los fundamentos epistemológicos respecto del liderazgo distribuido se encuentran en la teoría de la actividad y de la cognición distribuida. Derivadas de estas teorías se observan dos grandes discusiones conceptuales: la de Spillane et al. (2001), quienes se han basado en gran medida en la cognición distribuida para generar un liderazgo distribuido, y la de Gronn (2002) quien ha utilizado la teoría de la actividad para orientar su trabajo. Ambos enfoques son importantes como indicadores de los tipos de investigación que se pueden realizar para fortalecer el rendimiento de las formas y actividades del liderazgo como una actividad distribuida. 


\section{Teoría de la actividad}

Esta teoría ofrece un marco conceptual en el que se formulan nuevas unidades de análisis que relacionan al sujeto y su medio sociocultural. Una de estas unidades es la actividad, desarrollada por Leontiev a finales de los años 70 y reformulada por Engeström (1987).

La teoría de la actividad realiza un análisis del comportamiento y el conocimiento humano posicionado en un momento histórico, respecto de los sistemas de actividad orientados a un objetivo y sobre la base de la interacción cooperativa entre las personas. Al decir de Leontiev (1981), el sistema de actividad constituye una unidad básica para analizar los procesos psicológicos y sociales, tanto de la cultura como un todo, así como de los sujetos en forma individual.

Los sistemas de actividad se caracterizan por desarrollarse históricamente, estar mediados por herramientas, ser dialécticamente estructurados y analizarse desde la perspectiva de la relación entre los participantes y las herramientas utilizadas. De acuerdo con estos elementos, el cambio en el comportamiento humano no se produciría por sí solo, sino en la interacción con otros, ya que los sistemas de actividad son por naturaleza de carácter social. Leontiev (1981) señala que un sistema de actividad no resulta de la sumatoria de las reacciones entre los individuos, sino que se configura como un sistema con su propia estructura, sus procesos internos y desarrollo particular.

En relación con este enfoque, las actividades humanas son sistemas complejos que cambian en forma continua, interactúan y se reorganizan a medida que las personas se adaptan y producen transformaciones en el ambiente. En otras palabras, esto significa que el conocimiento no se produce en forma individual sino que es inter subjetivo, la actividad humana es social. El cambio no se produce en un único sentido, se genera a partir de una actividad conjunta, ya que los sistemas de actividad son dialécticos, sean éstos cooperativos, conflictivos, de frente o a través de redes virtuales. Según Engeström (1991) la unidad de análisis no resulta del trabajo individual, sino de la relación entre las personas que lo conforman.

Larripa y Erausquin (2008) señalan que la actividad histórico-cultural ha evolucionado, al menos en tres etapas en el desarrollo de las investigaciones:

1. La primera, se basa en la idea de mediación cultural, es decir, la acción humana es mediada por los instrumentos y se orienta hacia ciertos objetivos. Se produce una tríada: objeto, sujeto y artefacto mediador, rompiéndose el dualismo cartesiano de individuo y sociedad. La implicancia de esta mirada es que para entender al individuo no se puede prescindir de entender su medio cultural.

2. En la segunda etapa, los trabajos de Leontiev (1981) permiten centrarse en la actividad colectiva. En ese contexto Engeström (2001) señala que la actividad es una formación colectiva que responde a una compleja estructura mediadora. Esto significa que los sistemas de actividad no se limitan a acciones, con un inicio y un término, sino que evolucionan a través de un tiempo socio-histórico y adoptan la forma de instituciones y organizaciones. De esta forma, la comunidad, las reglas y la división del trabajo posibilitan el análisis de sus interacciones y de los sistemas de actividad a un nivel macro que considera lo colectivo y la comunidad, sin concentrarse sólo en el individuo.

3. Engeström (2001), en la última etapa, desarrolla el análisis de los procesos de aprendizaje interorganizacionales, sus tensiones, contradicciones, negociaciones y luchas entre los objetivos y las percepciones de los miembros de estos sistemas.

Para examinar el concepto de liderazgo distribuido Gronn (2002) utiliza la teoría de la actividad de Engeström (2000). Esta enfatiza la actividad realizada en conjunto, la centralidad de la división del trabajo, la fluidez de las relaciones, los grados de libertad abierta a actores sociales y la dinámica interna del sistema de actividad que permite la transformación a través de pequeños cambios. En definitiva, la teoría de la actividad podría resumirse como aquella que describe la vida social como un proceso de relaciones en constante movimiento entre las 
tecnologías, la naturaleza, las ideas (conceptos), personas y comunidades. Su foco de acción pasa de una persona a otra de acuerdo con el contexto social y medioambiental y con el flujo de acción dentro de éste. En efecto, un individuo puede iniciar el cambio, en conjunto con otros que lo siguen, le contribuyen, aportan o lo alteran de varias formas. Sus acciones tienen un efecto hacia atrás y alteran las condiciones, relaciones, reglas, etc. de ese contexto. En síntesis, el flujo de la actividad incluye la circulación de la iniciativa. En trabajos anteriores Gronn, deja claro que también está influenciado por la teoría sociológica del dualismo analítico de Archer.

\section{Teoría de la Cognición Distribuida}

Desarrollada por Hutchins en los años 80, plantea un nuevo paradigma que permite repensar los dominios de la cognición. En este sentido, Hutchins (1995) postula que la cognición debe ampliarse más allá de los procesos que le ocurren al individuo, para incluir otros sistemas técnicos sociales o sistemas cognitivos de mayor escala. Por consiguiente, la cognición distribuida es un proceso de construcción de significado que incorpora situación, acción y artefactos como un todo. Por ejemplo, el uso de una calculadora no se separa de la resolución de un problema matemático: es una parte integral de la búsqueda de la solución. La actividad cognitiva se extiende a los actores y los artefactos para dar sentido a la situación, por lo tanto, es importante reconocer la naturaleza versátil de cualquier situación. De esta forma las personas, la historia, los eventos y el entorno físico son todos partes de la situación en la que se ejerce el liderazgo. Una consecuencia de esto es que se puede ver el liderazgo como "coejecutado por" o "extendido a" los actores y los artefactos que se incorporan en el proceso y, quizás, ubicados "entre" los actores interdependientes. Otra consecuencia es que el liderazgo puede encontrarse tanto en cargos formales dentro de una organización como en relaciones informales que dependen de formas de experiencia particulares.

Los fundamentos de la discusión de Spillane et al. (2001) sobre el liderazgo distribuido están en los conceptos de cognición distribuida y teoría de la actividad. Esta perspectiva del liderazgo distribuido examina cómo las responsabilidades del liderazgo y las acciones a través de las cuales éstas son ejecutadas se distribuyen entre lo que llaman liderazgo posicional e informal. Esto requiere un análisis cuidadoso de dos cosas: a) la o las tareas incluidas dentro de su ubicación (responsabilidades del liderazgo), y b) la relación entre las macro y micro actividades y los procesos involucrados (las acciones a través de las cuales se ejerce el liderazgo). En otras palabras, esto conlleva el estudio de cómo se lleva a cabo y de qué es. A partir de esta perspectiva, los líderes escolares deben tomar parte en la definición de las tareas y en la ejecución de ellas, lo que requiere la utilización activa de las teorías defendidas por los individuos y las teorías en uso. Se debe entender, entonces, que el liderazgo se desarrolla desde la perspectiva de los profesionales y a través de sus teorías en uso.

\section{Revisión de estudios de liderazgo distribuido}

Con el propósito de entender el campo de estudio del liderazgo distribuido, se realizará a continuación un análisis de investigaciones publicadas en revistas y libros internacionales. Se utilizaron bases de datos bibliográficas para buscar los textos y se utilizó cada artículo o libro para pesquisar dentro de su bibliografía otras referencias pertinentes. Los trabajos que se presentan a continuación se agrupan según las siguientes características: autor/es y título del trabajo; conceptos y teoría-estrategias; metodológicas y técnicas utilizadas. 
Tabla 1. Principales características de las conceptualizaciones sobre el liderazgo distribuido

\begin{tabular}{|c|c|c|c|}
\hline $\begin{array}{c}\text { Autor/es y título del } \\
\text { trabajo }\end{array}$ & Conceptos y teorías & Estrategia metodológica & Técnicas utilizadas \\
\hline $\begin{array}{l}\text { Spillane, J.P., Halverson, } \\
\text { R. \& Diamond, J.B. } \\
\text { (2001) Investigating } \\
\text { school leadership } \\
\text { practice: a distributed } \\
\text { perspective. Educational } \\
\text { Researcher 30(3): 23-28. }\end{array}$ & $\begin{array}{l}\text { Los fundamentos de la } \\
\text { discusión sobre el liderazgo } \\
\text { distribuido están en los } \\
\text { conceptos de cognición } \\
\text { distribuida y teoría de la } \\
\text { actividad. } \\
\text { La cognición distribuida es un } \\
\text { proceso de construcción de } \\
\text { significado que incorpora } \\
\text { situación, acción y artefactos } \\
\text { como un todo. } \\
\text { La actividad cognitiva se } \\
\text { extiende a los actores y los } \\
\text { artefactos para dar sentido a la } \\
\text { situación, y es importante } \\
\text { reconocer la naturaleza versátil } \\
\text { de cualquier situación. } \\
\text { Los autores sostienen que a } \\
\text { partir de esta perspectiva, los } \\
\text { líderes escolares deben tomar } \\
\text { parte en la definición de las } \\
\text { tareas y en la ejecución de ellas, } \\
\text { y esta actividad requiere la } \\
\text { utilización activa de las teorías } \\
\text { defendidas por los individuos y } \\
\text { las teorías en uso. Se debe } \\
\text { entender, entonces, que el } \\
\text { liderazgo se desarrolla desde la } \\
\text { perspectiva de los profesionales } \\
\text { y a través de sus teorías en uso. }\end{array}$ & $\begin{array}{l}\text { Los autores realizan un } \\
\text { análisis cualitativo, y } \\
\text { recurren a tres ejemplos } \\
\text { para ilustrar el argumento } \\
\text { general del estudio. } \\
\text { 1. Observación de los } \\
\text { intentos que hace una } \\
\text { escuela primaria } \\
\text { centrando los esfuerzos } \\
\text { de mejora en base a las } \\
\text { necesidades de } \\
\text { aprendizajes de los } \\
\text { estudiantes. } \\
\text { 2. El director y miembros } \\
\text { del equipo directivo se } \\
\text { reúnen con cada profesor } \\
\text { para tratar la planificación. } \\
\text { 3. Sugerencias sobre } \\
\text { cómo la práctica del } \\
\text { liderazgo puede } \\
\text { extenderse a dos o más } \\
\text { individuos. }\end{array}$ & $\begin{array}{l}\text { Análisis de contenido } \\
\text { de las observaciones } \\
\text { de las prácticas de } \\
\text { liderazgo. }\end{array}$ \\
\hline $\begin{array}{l}\text { Gronn, P. (2002) } \\
\text { Distributed leadership. } \\
\text { In: K. Leithwood \& P. } \\
\text { Hallinger (eds). Second } \\
\text { International Handbook } \\
\text { of Educational } \\
\text { Leadership and } \\
\text { Administration. } \\
\text { Dordrecht: Kluwer, pp. } \\
653-696 .\end{array}$ & $\begin{array}{l}\text { En el trabajo se recurre a la } \\
\text { teoría de la actividad de } \\
\text { Engeström. También está } \\
\text { influenciado por la teoría } \\
\text { sociológica del dualismo } \\
\text { analítico. } \\
\text { Define el liderazgo como una } \\
\text { "influencia emergente } \\
\text { relacionada con el trabajo" } \\
\text { Un asunto clave es el } \\
\text { argumento de que el liderazgo } \\
\text { distribuido es empírico. }\end{array}$ & $\begin{array}{l}\text { El autor basa y sustenta su } \\
\text { teorización del liderazgo } \\
\text { distribuido usando como } \\
\text { referencia una variedad de } \\
\text { estudios empíricos de } \\
\text { investigadores en diversos } \\
\text { campos (meta análisis). } \\
\text { Desarrolla el concepto } \\
\text { como una herramienta } \\
\text { analítica para comprender } \\
\text { este aspecto creciente del } \\
\text { liderazgo en práctica. }\end{array}$ & Análisis de contenido \\
\hline
\end{tabular}

Fuente: Elaboración propia. 
En resumen, ambos autores presentan trabajos importantes como guías de los tipos de investigaciones que fortalecen nuestra comprensión de las formas de actividad de liderazgo que pueden ser consideradas como distribuidas. En ambos estudios los autores buscan defender que el liderazgo debe ser analizado en función de la práctica.

Tabla 2. Estudios estadísticos con datos empíricos

\begin{tabular}{|c|c|c|c|}
\hline $\begin{array}{c}\text { Autor/es y título del } \\
\text { trabajo }\end{array}$ & Conceptos y teorías & Estrategia metodológica & $\begin{array}{l}\text { Técnicas } \\
\text { utilizadas }\end{array}$ \\
\hline $\begin{array}{l}\text { Leithwood, K. \& Jantzi, } \\
\text { D. (1999). The relative } \\
\text { effects of principal and } \\
\text { teacher sources of } \\
\text { leadership on student } \\
\text { engagement with school. } \\
\text { Educational } \\
\text { Administration Quarterly } \\
\text { 35(5): 679-706. }\end{array}$ & $\begin{array}{l}\text { Su estudio se centra en } \\
\text { identificar empíricamente el } \\
\text { grado en que el "liderazgo del } \\
\text { profesor" puede considerarse } \\
\text { efectivo. } \\
\text { Los autores sugieren que el } \\
\text { liderazgo puede ser visto como } \\
\text { el ejercicio de la influencia: } \\
\text { como construido socialmente, } \\
\text { más que definido } \\
\text { estructuralmente en base al } \\
\text { cargo que un sujeto ocupa } \\
\text { formalmente en la } \\
\text { organización. }\end{array}$ & $\begin{array}{l}\text { Modelos causales, para } \\
\text { evaluar los efectos } \\
\text { directos e indirectos del } \\
\text { liderazgo del director y del } \\
\text { profesor en el } \\
\text { compromiso del } \\
\text { estudiante. }\end{array}$ & $\begin{array}{l}\text { Análisis factorial } \\
\text { exploratorio, } \\
\text { confirmatorios y } \\
\text { ecuaciones } \\
\text { estructurales }\end{array}$ \\
\hline $\begin{array}{l}\text { Heck, R. H. \& Hallinger, } \\
\text { P. (2009). Assessing the } \\
\text { contribution of } \\
\text { distributed leadership to } \\
\text { school improvement and } \\
\text { growth in math } \\
\text { achievement. American } \\
\text { Educational Research } \\
\text { Journal 46(3): 659-689. }\end{array}$ & $\begin{array}{l}\text { Los autores apoyan la } \\
\text { perspectiva del liderazgo } \\
\text { distribuido, que tiene como } \\
\text { objetivo la construcción de la } \\
\text { capacidad académica de las } \\
\text { escuelas como una forma de } \\
\text { mejorar los resultados de } \\
\text { aprendizaje de los estudiantes. }\end{array}$ & $\begin{array}{l}\text { Estudio longitudinal no } \\
\text { experimental, en el que se } \\
\text { pone a prueba un modelo } \\
\text { conceptual de los efectos } \\
\text { del liderazgo distribuido } \\
\text { en el aumento del } \\
\text { rendimiento en } \\
\text { matemáticas (análisis } \\
\text { multivariado). }\end{array}$ & $\begin{array}{l}\text { Análisis Multinivel. } \\
\text { Modelos de } \\
\text { ecuaciones } \\
\text { estructurales. }\end{array}$ \\
\hline $\begin{array}{l}\text { Spillane, J. P. \& Healey, } \\
\text { K. (2010). } \\
\text { Conceptualizing school } \\
\text { leadership and } \\
\text { management from a } \\
\text { distributed perspective: } \\
\text { an exploration of some } \\
\text { study operations and } \\
\text { measures. The } \\
\text { Elementary School } \\
\text { Journal } 111(2): 253-281 .\end{array}$ & $\begin{array}{l}\text { Para los autores una } \\
\text { perspectiva distribuida tiene el } \\
\text { potencial de } \\
\text { generar nuevos conocimientos } \\
\text { sobre el liderazgo y la gestión } \\
\text { de las escuelas. } \\
\text { Definen el liderazgo y la gestión } \\
\text { en términos de práctica lo que } \\
\text { permite la posibilidad de que } \\
\text { las personas sin designaciones } \\
\text { formales de liderazgo pueden } \\
\text { asumir responsabilidades de } \\
\text { trabajo en la organización. }\end{array}$ & $\begin{array}{l}\text { Los investigadores utilizan } \\
\text { datos de } 30 \text { escuelas } \\
\text { primarias urbanas de } \\
\text { tamaño medio, donde } \\
\text { analizan el grado en que } \\
\text { estas medidas recogen la } \\
\text { variación entre escuelas y } \\
\text { luego evalúan } \\
\text { críticamente los puntos } \\
\text { fuertes y débiles de las } \\
\text { muestras medidas. }\end{array}$ & $\begin{array}{l}\text { Estadística descriptiva; } \\
\text { correlaciones. }\end{array}$ \\
\hline $\begin{array}{l}\text { Baloglu, N. (2012) } \\
\text { Relations between } \\
\text { value-based leadership } \\
\text { and distributed }\end{array}$ & $\begin{array}{l}\text { El autor basa su estudio en } \\
\text { consideración con la teoría de } \\
\text { la actividad. } \\
\text { Para el autor, el liderazgo }\end{array}$ & Análisis Multivariado. & $\begin{array}{l}\text { Correlaciones } \\
\text { (Pearson), análisis } \\
\text { factorial confirmatorio } \\
\text { y regresión lineal }\end{array}$ \\
\hline
\end{tabular}




\begin{tabular}{|l|l|l|l|}
\hline leadership: a casual & distribuido significa que las & múltiple. \\
research on school & personas están trabajando en \\
principles' behaviors. & conjunto para poner en común & & \\
Educational Sciences: & sus iniciativas y experiencias, & & \\
Theory \& Practice $12(2)$ & por lo que el resultado es \\
Special Issue: $1375-1378$. & $\begin{array}{l}\text { mayor que la suma de sus } \\
\text { acciones individuales. }\end{array}$ & & \\
\hline
\end{tabular}

Fuente: Elaboración propia.

En resumen, en los estudios estadísticos revisados, la principal característica está dada por las técnicas de análisis de datos avanzadas que utilizan los diversos autores para dar respuesta a los objetivos planeados (regresión múltiple, análisis factoriales exploratorios y confirmatorios, ecuaciones estructurales y multinivel).

Tabla 3. Tipologías principales de estudios interculturales asociados al liderazgo distribuido

\begin{tabular}{|c|c|c|c|}
\hline $\begin{array}{c}\text { Autor/es y título del } \\
\text { trabajo }\end{array}$ & Conceptos y teorías & Estrategia metodológica & Técnicas utilizadas \\
\hline $\begin{array}{l}\text { Bryant, M. (2003) Cross- } \\
\text { cultural perspectives on } \\
\text { school leadership: } \\
\text { lessons from Native } \\
\text { American interviews. En: } \\
\text { N. Bennett, M. Crawford } \\
\text { \& M. Cartwright (eds.) } \\
\text { Effective Educational } \\
\text { Leadership. London: } \\
\text { Sage, pp. 216-228. }\end{array}$ & $\begin{array}{l}\text { Percepción de que el liderazgo } \\
\text { no está localizado en una } \\
\text { persona sino en una } \\
\text { comunidad, y que “líder" tiene } \\
\text { una posición momentánea. } \\
\text { Cada persona juega un rol de } \\
\text { liderazgo en diferentes } \\
\text { momentos, y puede hacer una } \\
\text { contribución significativa }\end{array}$ & Estudio de caso & $\begin{array}{l}\text { Entrevistas largas con } \\
\text { nativos americanos de } \\
\text { seis tribus diferentes }\end{array}$ \\
\hline $\begin{array}{l}\text { Chang, I. (2011). A study } \\
\text { of the relationships } \\
\text { between distributed } \\
\text { leadership, teacher } \\
\text { academic optimism and } \\
\text { student achievement in } \\
\text { Taiwanese elementary } \\
\text { schools. School } \\
\text { Leadership \& } \\
\text { Management 31(5): 491- } \\
515 \text {. }\end{array}$ & $\begin{array}{l}\text { El estudio se centró en las } \\
\text { escuelas primarias públicas en } \\
\text { Taiwán. } \\
\text { El autor piensa que el liderazgo } \\
\text { distribuido crea significado y } \\
\text { valor para la organización y que } \\
\text { la función del liderazgo se } \\
\text { dispersa entre todos los } \\
\text { miembros de la organización o } \\
\text { grupo con el propósito de } \\
\text { aumentar la conciencia de los } \\
\text { miembros para que la } \\
\text { organización cuente con más } \\
\text { líderes para lograr los objetivos. }\end{array}$ & $\begin{array}{l}\text { Se solicitó a los maestros } \\
\text { evaluar el papel de su } \\
\text { director en dirigir y } \\
\text { facilitar el liderazgo } \\
\text { distribuido en las escuelas. }\end{array}$ & $\begin{array}{l}\text { Cálculo de las } \\
\text { estadísticas } \\
\text { descriptivas (media, } \\
\text { desviación estándar, } \\
\text { asimetría y curtosis). } \\
\text { Análisis de varianza y } \\
\text { ecuaciones } \\
\text { estructurales (SEM). }\end{array}$ \\
\hline $\begin{array}{l}\text { Singh, M., Han, J. \& } \\
\text { Woodrow, C. (2012). } \\
\text { Shifting pedagogies } \\
\text { through distributed } \\
\text { leadership: mentoring } \\
\text { Chilean early childhood } \\
\text { educators in literacy } \\
\text { teaching. Australasian } \\
\text { Journal of Early }\end{array}$ & $\begin{array}{l}\text { El fenómeno investigado en } \\
\text { este estudio es el impacto de la } \\
\text { formación profesional de los } \\
\text { enfoques de liderazgos } \\
\text { pedagógicos distribuidos en el } \\
\text { liderazgo y la alfabetización de } \\
\text { los educadores de párvulos. } \\
\text { El liderazgo pedagógico }\end{array}$ & $\begin{array}{l}\text { Los autores utilizaron el } \\
\text { Programa Futuro Infantil } \\
\text { Hoy (PFIH). Programa de } \\
\text { aprendizaje profesional } \\
\text { para los educadores de } \\
\text { párvulos chilenos, } \\
\text { desarrollado por un } \\
\text { equipo de académicos } \\
\text { australianos expertos en }\end{array}$ & $\begin{array}{l}\text { Entrevistas con los } \\
\text { cuatro grupos de } \\
\text { informantes claves: } \\
\text { educadores/as de } \\
\text { párvulos, directores/as } \\
\text { de los centros, } \\
\text { sostenedores y las/os } \\
\text { asistentes de la } \\
\text { enseñanza. }\end{array}$ \\
\hline
\end{tabular}


Childhood 37(4): 68-76.

distribuido fue concebido como un vehículo para la aplicación de un enfoque socio-cultural a la lengua española y a la alfabetización.

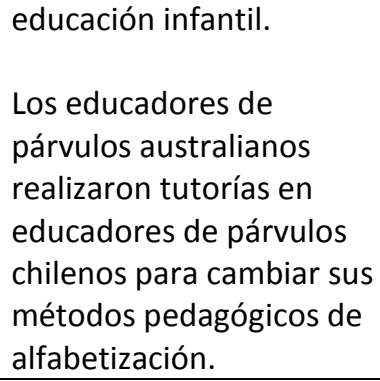

educación infantil.

Los educadores de párvulos australianos realizaron tutorías en educadores de párvulos chilenos para cambiar sus métodos pedagógicos de alfabetización.

Fuente: Elaboración propia.

En resumen, los estudios interculturales revisados son los siguientes: 1) experiencias con nativos americanos de seis tribus diferentes, 2) escuelas primarias públicas en Taiwán y 3) una comunidad de escolar preescolar al norte de Chile. En ellos está presente el liderazgo distribuido en el ámbito pedagógico. Es significativo destacar que en este tipo de estudios se utilizaron diversas técnicas tanto cualitativas (entrevista, observaciones, etc.) como cuantitativas (análisis de varianza, ecuaciones estructurales, etc.).

Tabla 4. Estudios de liderazgo distribuido que emergen de iniciativas o influencias dispersas

\begin{tabular}{|c|c|c|c|}
\hline $\begin{array}{c}\text { Autor/es y título del } \\
\text { trabajo }\end{array}$ & Conceptos y teorías & Estrategia metodológica & Técnicas utilizadas \\
\hline $\begin{array}{l}\text { Hartley, J. \& Allison, M. } \\
\text { (2000) The } \\
\text { modernization and } \\
\text { improvement of } \\
\text { government and public } \\
\text { services: the role of } \\
\text { leadership in the } \\
\text { modernization and } \\
\text { improvement of public } \\
\text { services. Public Money } \\
\text { and Management 20(2): } \\
35-40\end{array}$ & $\begin{array}{l}\text { Definen el liderazgo distribuido } \\
\text { como el resultado de alianzas y } \\
\text { trabajo en equipo y como la } \\
\text { consecuencia natural de nuevas } \\
\text { formas de trabajo cooperativo y } \\
\text { estructuras más horizontales. }\end{array}$ & Estudios de casos & $\begin{array}{l}\text { Entrevistas en } \\
\text { profundidad y grupos } \\
\text { focales con } \\
\text { empleados. } \\
\text { También se agregaron } \\
\text { observaciones y } \\
\text { documentación. }\end{array}$ \\
\hline $\begin{array}{l}\text { Harris, A. \& Chapman, C. } \\
\text { (2002). Democratic } \\
\text { leadership for school } \\
\text { improvement in } \\
\text { challenging contexts. } \\
\text { International Electronic } \\
\text { Journal for Leadership in } \\
\text { Learning, } 6(9): 1-9 .\end{array}$ & $\begin{array}{l}\text { En esta descripción, el liderazgo } \\
\text { democrático incluye el } \\
\text { liderazgo distribuido, este } \\
\text { último consiste en un proceso } \\
\text { en el que se delega } \\
\text { responsabilidad y autoridad a } \\
\text { grupos de administradores } \\
\text { sénior y, luego, en un nivel más } \\
\text { amplio, se da a los profesores la } \\
\text { oportunidad de compartir la } \\
\text { toma de decisiones, de sacar lo } \\
\text { mejor a través de estas } \\
\text { estrategias y otorgar } \\
\text { reconocimiento. }\end{array}$ & Estudio de caso & $\begin{array}{l}\text { Entrevistas } \\
\text { semiestructuradas con } \\
\text { directores, gerentes } \\
\text { medios y profesores } \\
\text { de aula, además de } \\
\text { evaluaciones de las } \\
\text { conclusiones con otros } \\
\text { directivos, y una } \\
\text { recolección de datos } \\
\text { de documentación. }\end{array}$ \\
\hline $\begin{array}{l}\text { Oborn, E., Barrett, M. \& } \\
\text { Dawson, S. (2013) } \\
\text { Distributed leadership in }\end{array}$ & $\begin{array}{l}\text { Los autores utilizan el concepto } \\
\text { de liderazgo distribuido desde } \\
\text { un enfoque sociomaterial para }\end{array}$ & $\begin{array}{l}\text { Estudios de casos } \\
\text { (etnografía política) }\end{array}$ & $\begin{array}{l}\text { Observaciones en } \\
\text { reuniones, } \\
\text { presentaciones, }\end{array}$ \\
\hline
\end{tabular}




\begin{tabular}{|c|c|c|}
\hline $\begin{array}{l}\text { policy formulation: a } \\
\text { sociomaterial } \\
\text { perspective. } \\
\text { Organization Studies } \\
34(2): 253-276\end{array}$ & $\begin{array}{l}\text { revelar cómo y por qué el } \\
\text { liderazgo se distribuye a través } \\
\text { de sus prácticas que, en } \\
\text { conjunto, (re) configuran } \\
\text { coaliciones de políticas del } \\
\text { contexto. }\end{array}$ & $\begin{array}{l}\text { conferencias, eventos } \\
\text { y encuentros } \\
\text { informales que } \\
\text { suscitaron debates, } \\
\text { opiniones y } \\
\text { experiencias de } \\
\text { diversas personas. } \\
\text { Grupos de discusión } \\
\text { del equipo de política. } \\
\text { Entrevistas, notas } \\
\text { etnográficas detalladas } \\
\text { y transcripciones de } \\
\text { participación pública. }\end{array}$ \\
\hline
\end{tabular}

Fuente: Elaboración propia.

En resumen, estos artículos presentan ambigüedades en la definición del liderazgo distribuido. También han dado lugar a una diversa nomenclatura que se utiliza en la literatura, tales como el liderazgo democrático (Woods 2004) y el liderazgo compartido (Pearce y Conger 2003). Estos términos son utilizados con frecuencia, por ejemplo, "liderazgo distribuido" y "liderazgo compartido" se utilizan a menudo en el mismo papel como si fueran iguales. El uso de estos conceptos crea confusión en la aplicación práctica de las definiciones y plantea dificultades de interpretación teniendo en cuenta las implicancias de los hallazgos basados en estudios de investigación.

Tabla 5. Estudios que tratan conceptos de liderazgo relacionados con el liderazgo distribuido, y que tienen implicaciones para su comprensión, pero que no se refieren explícitamente al liderazgo distribuido

\begin{tabular}{|c|c|c|c|}
\hline $\begin{array}{c}\text { Autor/es y título del } \\
\text { trabajo }\end{array}$ & Conceptos y teorías & Estrategia metodológica & Técnicas utilizadas \\
\hline $\begin{array}{l}\text { Knight, P. T. \& Trowler, } \\
\text { P. R. (2001) } \\
\text { Departmental leadership } \\
\text { in higher education. } \\
\text { Buckingham: SRHE/Open } \\
\text { University. }\end{array}$ & $\begin{array}{l}\text { Los autores apoyan hasta cierto } \\
\text { punto las teorías de la actividad } \\
\text { social de Wenger y Engeström, } \\
\text { asociándolas con las teorías de } \\
\text { Vygotsky sobre el aprendizaje y } \\
\text { el compromiso social con el } \\
\text { mundo como lo presenta } \\
\text { Wertsch. }\end{array}$ & Análisis de contenido & $\begin{array}{l}\text { Entrevistas vía web y } \\
\text { correo electrónico con } \\
\text { líderes académicos }\end{array}$ \\
\hline $\begin{array}{l}\text { Gold, A. (2003) } \\
\text { Principled principals? } \\
\text { Values-driven } \\
\text { leadership: evidence } \\
\text { from ten case studies of } \\
\text { 'outstanding' school } \\
\text { leaders. Educational } \\
\text { Management } \\
\text { Administration \& } \\
\text { Leadership 31(2): 127- } \\
138\end{array}$ & $\begin{array}{l}\text { Los autores no utilizan el } \\
\text { término liderazgo distribuido, } \\
\text { en su lugar se refieren al } \\
\text { "desarrollo de la capacidad de } \\
\text { liderazgo" en cuanto involucra } \\
\text { la delegación de poder y } \\
\text { responsabilidad a directivos de } \\
\text { departamentos y facultades y } \\
\text { los involucra a ellos en grupos e } \\
\text { iniciativas de trabajo. }\end{array}$ & Estudios de casos & $\begin{array}{l}\text { Observaciones } \\
\text { etnográficas }\end{array}$ \\
\hline $\begin{array}{l}\text { Bolden, R., Petrov, G. \& } \\
\text { Gosling, J. (2008) }\end{array}$ & $\begin{array}{l}\text { Para los autores, las formas } \\
\text { resultantes de liderazgo en }\end{array}$ & $\begin{array}{l}\text { Se utiliza un modelo Multi- } \\
\text { Level con información }\end{array}$ & $\begin{array}{l}\text { Entrevistas semi- } \\
\text { estructuradas cara a }\end{array}$ \\
\hline
\end{tabular}




\begin{tabular}{|c|c|c|c|}
\hline $\begin{array}{l}\text { Tensions in higher } \\
\text { education leadership: } \\
\text { towards a multi-level } \\
\text { model of leadership } \\
\text { practice. Higher } \\
\text { Education Quarterly } \\
62(4): 358-376 .\end{array}$ & $\begin{array}{l}\text { educación están ampliamente } \\
\text { distribuidas (a través de las } \\
\text { personas, estructuras, redes). } \\
\text { Las formas resultantes de } \\
\text { liderazgo se pueden describir } \\
\text { más exactamente como } \\
\text { "híbridas" (que comprenden } \\
\text { tanto los enfoques } \\
\text { individualistas y colectivos). }\end{array}$ & $\begin{array}{l}\text { cualitativa, donde se } \\
\text { identifican los elementos } \\
\text { constitutivos de la práctica } \\
\text { del liderazgo en la } \\
\text { educación superior en los } \\
\text { niveles personal, social, } \\
\text { estructural, contextual y } \\
\text { de desarrollo. }\end{array}$ & $\begin{array}{l}\text { cara en } 12 \\
\text { universidades del } \\
\text { Reino Unido. } \\
\text { Además la } \\
\text { investigación utilizó la } \\
\text { técnica de la entrevista } \\
\text { en profundidad y } \\
\text { grupos de discusión } \\
\text { con los representantes } \\
\text { de la formación del } \\
\text { personal de las } \\
\text { instituciones } \\
\text { participantes. }\end{array}$ \\
\hline $\begin{array}{l}\text { Lopez, V., Ahumada, L., } \\
\text { Galdames, S. \& Madrid, } \\
\text { R. (2012) } \\
\text { School principals at their } \\
\text { lonely work: recording } \\
\text { workday practices } \\
\text { through ESM logs. } \\
\text { Computers \& Education } \\
\text { 58(1): 413-422 }\end{array}$ & $\begin{array}{l}\text { Este estudio trata de ofrecer } \\
\text { una herramienta para la } \\
\text { investigación del liderazgo } \\
\text { distribuido. } \\
\text { Conceptualmente, se aparta de } \\
\text { las opiniones tradicionales } \\
\text { sobre el líder y el enfoque } \\
\text { natural, ya que los autores no } \\
\text { están interesados en el líder } \\
\text { como persona individual, sino } \\
\text { en el patrón de interacciones } \\
\text { entre sujetos que realizan } \\
\text { tareas dentro del amplio } \\
\text { ámbito de la gestión escolar. }\end{array}$ & $\begin{array}{l}\text { Los autores utilizan un } \\
\text { diseño de métodos mixtos } \\
\text { secuenciales. } \\
\text { La investigación se realizó } \\
\text { en dos etapas: } \\
\text { En la primera fase, se ha } \\
\text { diseñado y implementado } \\
\text { un sistema de registro de } \\
\text { las experiencias } \\
\text { cotidianas. } \\
\text { En la segunda fase, se } \\
\text { realizó un seguimiento de } \\
\text { las entrevistas semi- } \\
\text { estructuradas con los } \\
\text { participantes de la primera } \\
\text { fase, en la que } \\
\text { comparten y discuten los } \\
\text { resultados primarios con el } \\
\text { fin de lograr la } \\
\text { comprensión en } \\
\text { profundidad de los datos. }\end{array}$ & $\begin{array}{l}\text { Registros de } \\
\text { experiencias cotidianas } \\
\text { Entrevistas } \\
\text { semiestructuradas }\end{array}$ \\
\hline
\end{tabular}

Fuente: Elaboración propia.

En resumen, de estos artículos podemos enfatizar el trabajo de Bolden, Petrov y Gosling por la descripción del liderazgo como "híbrido". En dicho trabajo se vinculan los enfoques individualistas y colectivos de acuerdo con Gronn y, además, se utiliza una estrategia metodológica novedosa, pues se realiza un estudio multinivel con información cualitativa en los niveles personal, social, estructural, contextual y de desarrollo.

\section{Análisis}

De acuerdo al análisis realizado puede inferirse que, conforme a los estudios revisados, los fundamentos de la discusión sobre el liderazgo distribuido se encuentran principalmente en los conceptos de cognición distribuida y teoría de la actividad. Sin embargo, hay autores como Gronn (2002) que en sus análisis también fueron influenciados por la teoría sociológica del dualismo analítico. 
En relación con las discusiones conceptuales sobre el liderazgo distribuido, Spillane et al. (2001) presenta una pequeña cantidad de datos en forma de tres ilustraciones obtenidas a partir de su investigación, mientras que Gronn (2002) realiza varios estudios para probar la utilidad de su teorización del término. Estos dos trabajos son de gran relevancia y fortalecen la comprensión conceptual del liderazgo distribuido. El aspecto más importante a destacar es que el análisis bibliográfico muestra que numerosos autores contextualizan sus investigaciones en función de los trabajos propuestos por Spillane y Gronn.

Por otro lado, la mayoría de las investigaciones empíricas examinadas son "estudios de casos" cualitativos en entornos organizacionales educativos. En esta dirección es importante reconocer que una variedad de ellos, en pequeña progresión, pueden construir un conjunto substancial de evidencias en el tiempo. Sin embargo, el número de investigaciones pertinentes sigue siendo reducido y nuevamente la evidencia que ellos generan debe ser juzgada como indicativa más que concluyente. Sin perjuicio de lo anterior, se destaca que un estudio de grado menor podría permitir develar estructuras profundas del fenómeno.

La fortaleza y solidez de los trabajos resumidos en este artículo deben ser consideradas como un conjunto de propuestas sobre la naturaleza del liderazgo distribuido y algunos posibles lineamientos para futuras investigaciones que exploren y validen la teoría más a fondo.

En las investigaciones revisadas fue difícil comprobar la estrategia metodológica utilizada por los autores, debido principalmente a que éstos generalmente no la explicitan en su diseño metodológico. Asimismo, encontramos que existe una importante cantidad de trabajos en que los autores, al no explicitar la estrategia metodológica, generan confusión con respecto de las técnicas de análisis que se utilizan, sobre todo en estudios de tipo cualitativo.

Finalmente debemos estar conscientes que las percepciones sociales y políticas del liderazgo distribuido contribuyen fuertemente al desarrollo de las organizaciones, preferentemente educativas. El liderazgo distribuido en contextos educativos promueve cambios en la cultura de la organización, acrecentando procesos de participación, democratización y toma de decisiones compartidas que inciden en el desarrollo de toda la comunidad escolar. La visión del liderazgo distribuido sobrepasa a la posición del liderazgo formal (directivos y miembros de los equipos de gestión) e incorpora las actividades de varias personas en una comunidad educativa que trabaja en la movilización y orientación del personal en los procesos de cambio.

Dada esta argumentación, si las investigaciones en educación y liderazgo distribuido se consolidan, tiene mucho sentido un análisis epistemológico de las bases teóricas y metodológicas de tal línea de investigación en ciencias sociales, pues su aplicación en el campo de la educación es de vital importancia en estos tiempos.

\section{Nota}

Este trabajo se inscribe dentro del desarrollo teórico preliminar en torno a la noción de liderazgo distribuido del proyecto FONDECYT 1130161. En este contexto, agradezco a todas(os) quienes aportaron a su concepción y desarrollo, como a todas(os) aquellas(os) que contribuyeron desde sus evaluaciones formales e informales.

\section{Bibliografía}

Bolden, R., Petrov, G. \& Gosling, J. 2008. Tensions in higher education leadership: towards a multi-level model of leadership practice. Higher Education Quarterly 62(4): 358-376. doi: 10.1111/j.1468-2273.2008.00398.x

Baloglu, N. 2012. Relations between value-based leadership and distributed leadership: a casual research on school principles' behaviors. Educational Sciences: Theory \& Practice 12(2), special issue: 1375-1378. 
Bryant, M. 2003. Cross-cultural perspectives on school leadership: lessons from Native American interviews. En: N. Bennett, M. Crawford \& M. Cartwright (eds.) Effective Educational Leadership. London: Sage, pp. 216-228.

Chang, I. 2011. A study of the relationships between distributed leadership, teacher academic optimism and student achievement in Taiwanese elementary schools. School Leadership \& Management 31(5): $491-515$. doi:10.1080/13632434.2011.614945

Engeström, Y. 1987. Learning by expanding: an activity-theoretical approach to developmental research. Helsinki: Orienta-Konsultit.

Engeström, Y. 1991. Non scolae sed vitae discimus: toward overcoming the encapsulation of school learning. Learning and Instruction 1(3): 243-259.

Engeström, Y. 2000. Activity theory and the social construction of knowledge: a story of four umpires. Organization 7(2): 301-310. doi:10.1177/135050840072006

Engeström, Y. 2001. Los estudios evolutivos del trabajo como punto de referencia de la teoría de la actividad: el caso de la práctica médica de la asistencia básica. En: S. Chaiklin y J. Lave (comps.). Estudiar las prácticas: perspectivas sobre actividad y contexto. Buenos Aires: Amorrortu Editores, pp. 78-118.

Gold, A. 2003. Principled principals? Values-driven leadership: evidence from ten case studies of 'outstanding' school leaders. Educational Management Administration \& Leadership 31(2): 127-138. doi: 10.1177/0263211X030312002

Gronn, P. 2002. Distributed leadership. En: K. Leithwood \& P. Hallinger (eds). Second International Handbook of Educational Leadership and Administration. Dordrecht: Kluwer, pp. 653-696.

Harris, A. \& Chapman, C. (2002). Democratic leadership for school improvement in challenging contexts. International Electronic Journal for Leadership in Learning 6(9): 1-9. http://eprints.gla.ac.uk/75099/

Hartley, J. \& Allison, M. 2000. The modernization and improvement of government and public services: the role of leadership in the modernization and improvement of public services. Public Money and Management 20(2): 35-40. doi:10.1111/1467-9302.00209

Heck, R. H. \& Hallinger, P. (2009). Assessing the contribution of distributed leadership to school improvement and growth in math achievement. American Educational Research Journal 46(3): 659-689. doi:10.3102/0002831209340042

Hutchins, E. 1995. How a cockpit remembers its speed. Cognitive Science 19(3): 265-288.

Knight, P.T. \& Trowler, P.R. 2001. Departmental leadership in higher education. Buckingham: SRHE/Open University.

Larripa, M. y Erausquin, C. 2008. Teoría de la actividad y modelos mentales. Instrumentos para la reflexión sobre la práctica profesional: "Aprendizaje expansivo", intercambio cognitivo y transformación de intervenciones de psicólogos y otros agentes en escenarios educativos. Anuario de Investigaciones de la Facultad de Psicología de la Universidad de Buenos Aires Vol. XV: 109-124.

Leithwood, K. \& Jantzi, D. 1999. The relative effects of principal and teacher sources of leadership on student engagement with school. Educational Administration Quarterly 35(5): 679-706.

Leontiev, A.N. 1981. Problems of the development of the mind. Moscow: Progress Publishers. 
Lopez, V; Ahumada, L; Galdames, S. \& Madrid, R. 2012. School principals at their lonely work: recording workday practices through through ESM logs. Computers \& Education 58(1): 413-422.

Oborn, E., Barrett, M. \& Dawson, S. 2013. Distributed leadership in policy formulation: a sociomaterial perspective. Organization Studies 34(2): 253-276. doi:10.1177/0170840612473552

Pearce, C.L. \& Conger, J.A. 2003. All those years ago: The historical underpinnings of shared leadership. In: C.L. Pearce \& J.A. Conger (eds) Shared leadership: reframing the hows and whys of leadership. Thousand Oaks, CA: SAGE, pp. 1-18.

Singh, M., Han, J. \& Woodrow, C. 2012. Shifting pedagogies through distributed leadership: mentoring Chilean early childhood educators in literacy teaching. Australasian Journal of Early Childhood 37(4): 68-76.

Spillane, J. P. \& Healey, K. 2010. Conceptualizing school leadership and management from a distributed perspective: an exploration of some study operations and measures. The Elementary School Journal 111(2): 253281. doi: $10.1086 / 656300$

Spillane, J. P., Halverson, R. \& Diamond, J.B. 2001. Investigating school leadership practice: a distributed perspective. Educational Researcher 30(3): 23-28. doi:10.3102/0013189X030003023

Woods, P. 2004. Democratic leadership: drawing distinctions with distributed leadership. International Journal of Leadership in Education 7(1): 3-26.

Recibido el 4 Mar 2013

Aceptado el 23 Jun 2013 